\title{
Gesture Friendly Interfaces for Classroom Teaching with Thinking Tools
}

\author{
Stephen Chen', George Frempong ${ }^{2}$, and William C. Cudmore ${ }^{3}$ \\ 1 School of Analytic Studies and Information Technology, York \\ University, 4700 Keele Street, Toronto, Ontario M3J 1P3 \\ sychen@yorku.ca \\ WWW home page: http://www.atkinson.yorku.ca/ sychen \\ 2 Faculty of Education, York University, \\ 4700 Keele Street, Toronto, Ontario M3J 1P3 \\ gfrempong@edu.yorku.ca \\ 3 School of Applied Computing and Engineering Sciences, Sheridan \\ College, 7899 McLaughlin Road, Brampton, Ontario L6V 1G6 \\ william.cudmore@sheridaninstitute.ca
}

\begin{abstract}
Thinking tools are typically designed for students working on their own computers. When used from the front of a classroom, the complex graphical user interfaces of these tools can interfere with an instructor's ability to lecture effectively and to use natural gestures. A thinking tool for the grade 9 mathematics topic of "relationships" has been developed with a more gesture-friendly interface. This gesture-friendly interface allows a teacher to focus more on interacting with students, creating engaging visualizations, and using natural hand and arm gestures as part of the lecture.
\end{abstract}

\section{Introduction}

The design of thinking tools has primarily been done from the perspective of the student. Designers have considered many issues such as determining what would help a student learn the material and how the student would want to interact with the new concepts. Although this student perspective is clearly important, it has largely occluded another important set of issues connected with the teacher perspective. A tool that is designed for individual students to use on their own computers may not be suitable for a teacher to use from the front of a classroom.

From the student's perspective, it is important for thinking tools to have an interactive component. To engage the student and to encourage independent inquiry, a student must be able to interact with and receive feedback from the tool. A 
common form of feedback is to see how a change in one model effects changes in another model [1]. For example, a tool may try to exploit a student's intuitive understanding of a physical model such as riding an escalator to help develop the student's understanding of an abstract mathematical concept such as a line equation. By being able to change a variable in one representation (e.g. height), the student will be able to see the effects in the other representation (e.g. y-coordinate).

From the teacher's perspective, an interface that allows rich student interactions will likely be quite difficult to use from the front of a classroom. In fact, a design goal for a thinking tool is to fully engage a (student) user in its operation and interactions. In a classroom environment that has a wall-sized screen and an instructor's PC located in a discreet corner of the room, the concentration required to manipulate the fine interface controls of the tool can absorb so much of the teacher's focus that they become detached from the classroom screen and their students. This disconnect can negatively affect the attentiveness of the students and the clarity of the teacher's presentation.

When presenting new material or making the first demonstration of the tool to the students it can be useful to focus the students' attention onto one aspect of the tool at a time. For example, a teacher may first want to demonstrate how the height of a passenger riding an escalator changes with time, and then demonstrate how the $y$-coordinate of a line equation changes with time. To identify one of two objects in normal, face-to-face conversations, people will often point [3]. However, if the teacher's hands and visual attention are fully engaged in operating the tool, he/she will be unable to point and to convey ideas through gestures. Without the ability to use gestures, speakers often compensate by using more complex descriptions [3].

Complex descriptions can imply complex concepts, so it may not be highly effective to use existing thinking tools as classroom teaching tools. A gesturefriendly interface (GFI) provides a "classroom mode" in which the control of a key visual component of the thinking tool is bound to a simple action such as pressing the space bar. With a GFI, a teacher can more easily focus on the students and use a free arm to perform natural gestures during a classroom presentation.

\section{Background}

Gesture-friendly interfaces are primarily intended for thinking tools which provide students with a significant opportunity for interaction and self-inquiry. As dynamic, interactive components, thinking tools are a critical component of learning objects that have been successful for more complex mathematical concepts [2][11]. A recommended design element for thinking tools is to have multiple representations with "dyna-linking" [1] so that each model moves in tandem regardless of which model is being manipulated. By interacting with these models, a student can, for example, use intuitive knowledge of a physical model to build a deeper understanding of the underlying abstract mathematical model.

From this research on design, there is also a growing awareness that "a prescriptive taxonomy and framework" [11, p158] is required to help match education objectives with the most suitable information visualization technique. For example, extensions to MathWorlds [10] include physical movements because

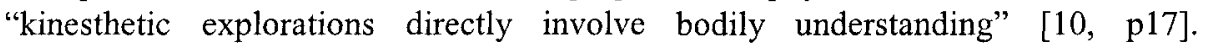


However, one of the interface components of MathWorlds uses button clicks to step through a time sequence - a gesture that few people will make with their hands when they are thinking or talking about events through time.

Physical ideas (such as movement) are actually embodied in abstract mathematical concepts through the use of fictive motion [7]. Subsequently, language alone cannot independently capture all of the dynamic aspects of some mathematical ideas. When directly interacting with the computer, the idea of fictive motion implies that click-and-drag sliders may be more effective than button clicks to "move" (a dynamic concept) down a number line (a static concept).

Using sliders and other more engaging interface components will not necessarily add any benefits to a classroom presentation. Students will neither perform the motion nor see it being performed, so they may miss key elements of the knowledge objectification process that were not communicated aurally by the teacher [9]. Further, the manipulation of sliders takes more concentration than the clicking of buttons, so the teacher's classroom lecture can suffer an additional distraction due to this design decision that primarily considers student usage.

If a teacher loses the ability to use gestures, he/she may have other cognitive functions impeded such as speech production [4], or at the least, will likely have to compensate with more complex descriptions of concepts that would normally be conveyed by gesture [3]. These side effects caused by the loss of gesture can negatively affect a teacher's ability to present the material, a student's absorption of the material, and a teacher's interest in using thinking tools. Overall, teachers are still most comfortable with technologies that facilitate "face-to-face whole class teaching" [8]. The goal of gesture-friendly interfaces is to take existing educational tools and make them more suitable for classroom teaching.

\section{Thinking tool design}

For the topic of "relationships" in the Ontario curriculum for mathematics [6], a learning object has been designed around the situation of choosing a cell phone plan. In this example, there are three available cell phone plans that can each have different fixed monthly service charges (i.e. y-intercept) and per minute rates (i.e. slope). The thinking tool embedded in the learning object allows students to analyze the effects of changing the monthly service charges, the per minute rates, and the minutes used (see Figure 1). These real-world components have been selected to match the key (abstract) concepts of intercepts, slopes, and line intersections.

The developed thinking tool uses dyna-linking [1] to connect the graphical and mathematical representations of the concept. With this linking, students can examine how changes to the (more abstract) line equations can affect the visually more concrete lines on the graph, and vice-versa. For example, clicking and dragging the left end-point of a line on the graph will simultaneously change the y-intercept in the corresponding line equation. Also, increasing or decreasing the slope in a line equation will simultaneously "rotate" the corresponding line on the graph. These linked, interactive components can help build a student's intuition on the concepts that connect the two representations. 


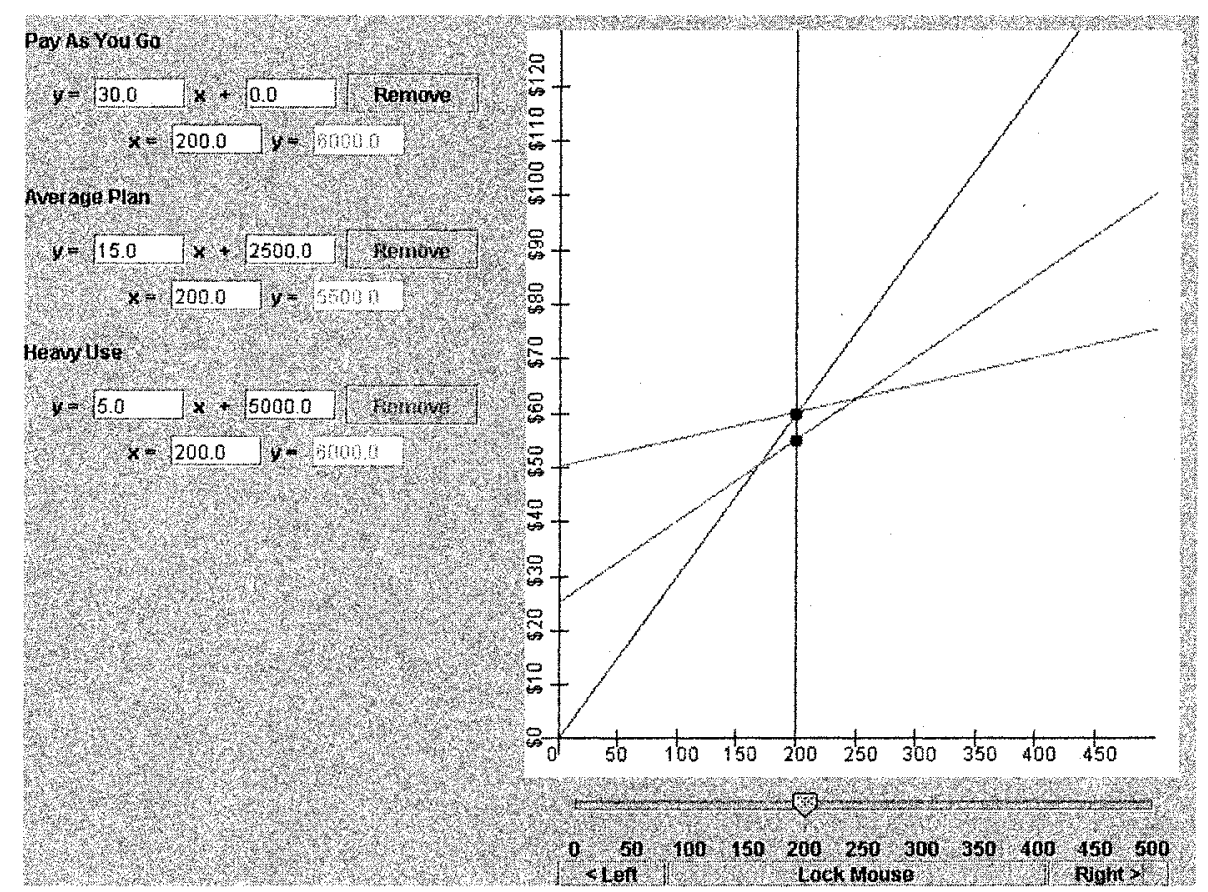

Figure 5. To activate the thinking tool's "classroom mode", the instructor can press the "Lock Mouse" button. When activated, the gesture-friendly interface allows left-button and rightbutton mouse clicks (on the graph) to increase or decrease the number of minutes to the next multiple of 50 minutes, or to the next line intersection if it is less than 50 minutes away.

\section{Gesture-friendly interface design}

To develop a GFI, the key feature of a classroom presentation must be determined. With the cell phone plans, a practical question that students will be familiar with is to determine which is the best plan (i.e. least expensive). The answer to this question depends on the number of minutes, and by varying the minutes, a teacher can show how different plans can be better for different amounts of usage. It can then be shown that the best plan changes at a line intersection, and that the location of line intersections depends on the monthly service charges ( $y$-intercepts) and per minute rates (slopes) of the cell phone plans (graphical lines and line equations).

A classroom presentation of the above material requires that the number of minutes can be easily varied by the teacher. If the teacher is using the slider, then his/her attention will be on his/her own PC's screen, and it will be difficult to point out the key features that the students should observe on the classroom screen. To make the developed thinking tool more suitable for classroom presentations, the interface component of left and right mouse button clicks is used because it is more gesture friendly than the standard GUI slider. Specifically, it is much easier to simultaneously click a button with one hand and gesture with the other hand than it is to click and drag and gesture at the same time. With a GFI, a teacher should be able to incorporate gestures into the lecture more easily, and this will enable them to interact with the classroom screen and the students more naturally and effectively. 


\section{Summary}

It is important to acknowledge that many teachers are more comfortable (and more effective) with face-to-face whole class teaching [8]. Face-to-face whole class teaching benefits from multi-modal forms of communication such as strong visualizations, physical embodiment of ideas, and dynamic interaction. To facilitate these modes of communication, educational technologies require easier control interfaces that are designed for classroom use. Gesture-friendly interfaces are a lowcost and technically trivial solution to this problem that can be supported by the existing technical infrastructure in a typical (Ontario) classroom.

\section{References}

1. Ainsworth, S. (1999) "The functions of multiple representations." Computers \& Education 33:131-152.

2. Gadanidis, G., Sedig, K., and Liang, H.N. (2004) "Designing online mathematical investigation." Journal of Computers in Mathematics and Science Teaching 23:273-296.

3. Kranstedt, K., Kühnlein, P., and Wachsmuth, I. (2004) "Deixis in multimodal human computer interaction: an interdisciplinary approach." In Camurri, A. and Volpe, G. (eds), Lecture Notes in Artificial Intelligence, Vol. 2915: Gesture-Based Communication in Human-Computer Interaction. 112-123.

4. Mayberry, R.I. and Jaques, J. (2000) "Gesture production during stuttered speech: Insights into the nature of gesture-speech integration." In McNeill, D. (ed) Language and Gesture: Window into Thought and Action. pgs 199-213.

5. Merill, M.D. (2000) "Knowledge Objects and Mental Models." In Proceedings of the International Workshop on Advanced Learning Technologies, 2000. pgs 244-246.

6. Ministry of Ed. and Training. (1999) The Ontario Curriculum, Grades 9 and 10: Math.

7. Núñez, R. (2004) "Do Real Numbers Really Move? Language, Thought, and Gesture: The Embodied Cognitive Foundations of Mathematics:" In Iida, F., et al (eds) Lecture Notes in Artificial Intelligence, Vol. 3139: Embodied Artificial Intelligence. pgs 54-73.

8. O'Malley, C. and Fraser, D.S. (2004) "Report 12: Literature Review in Learning with Tangible Technologies." A Report for NESTA Futurelab.

9. Radford, L. (2005). "Why do gestures matter? Gestures as semiotic means of Objectification." In Proceedings of the 29th Conference of the International Group for the Psychology of Mathematics Education, Vol. 1.pgs 143-145.

10. Roschelle, J.M., Kaput, J.J., and Stroup, W. "SimCalc: Accelerating students' engagement with the mathematics of change. In Jacobson, M. and Kozma, R. (eds) Innovations in science and mathematics education: Advanced designs for technologies of learning. 47-75.

11.Sedig, K., Rowhani, S., Morey, J., and Liang, H.N. (2003) "Application of information visualization techniques to the design of a mathematical mindtool: a usability study." Information Visualization 2:142-159. 